\title{
Occipital horn syndrome and classical Menkes Syndrome caused by deep intronic mutations, leading to the activation of ATP7A pseudo-exon
}

\author{
Saiqa Yasmeen ${ }^{1}$, Katrine Lund ${ }^{1}$, Anne De Paepe ${ }^{2}$, Sylvia De Bie ${ }^{2}$, Arvid Heiberg ${ }^{3}$, João Silva ${ }^{4}$, \\ Márcia Martins ${ }^{5}$, Tina Skjørringe ${ }^{1}$ and Lisbeth B Møller ${ }^{\star, 1}$
}

\begin{abstract}
Menkes disease is an X-linked disorder of copper metabolism caused by mutations in the ATP7A gene. Whereas most of the patients exhibit a severe classical form, about $9 \%$ of the patients exhibit a milder form of Menkes disease. The mildest form is called occipital horn syndrome (OHS). Mutations in the ATP7A gene can be identified in 95-98\% of the Menkes disease patients by standard screening techniques. Investigation of RNA isolated from the fibroblasts of eleven patients with no identified mutations was performed, and revealed inclusion of new pseudo-exons into the ATP7A mRNA from three unrelated patients: two patients with OHS and one patient with classical Menkes disease. The pseudo-exons were inserted between exons 10 and 11, between exons 16 and 17 and between exons 14 and 15 in the three patients, as a result of deep intronic mutations. This is the first time the activation of pseudo-exons is demonstrated in the ATP7A gene, and it demonstrates the usefulness of RNA analysis, in terms of revealing disease-causing mutations in noncoding regions. The fact that three different mutations cause disease by the activation of pseudo-exon inclusion also indicates that in Menkes disease this is an important mechanism, which has hitherto been overlooked.
\end{abstract}

European Journal of Human Genetics (2014) 22, 517-521; doi:10.1038/ejhg.2013.191; published online 4 September 2013

Keywords: Menkes disease; pseudo-exon; diagnosis; occipital horn syndrome; ATP7A

\section{INTRODUCTION}

Menkes disease (MD; OMIM\#309400) and occipital horn syndrome (OHS; OMIM\#304150) are allelic, X-linked recessive disorders of impaired copper metabolism due to mutations in the ATP7A gene. The patients with classical MD have severe developmental and neurological impairments due to subnormal amount of copper in the brain and a variety of symptoms such as connective tissue abnormalities, tortuosity of blood vessels and peculiar hair (kinky, steely hair or pili torti). ${ }^{1,2}$ Most of the classical MD patients die before the age of 3 years. The neurological symptoms of OHS patients are milder and lead to a clinical picture mainly characterized by connective tissue manifestations and skeletal abnormalities that include occipital exostoses, which give rise to the syndrome's name. ${ }^{3}$ These patients have normal or close-to-normal cognitive functions. The majority of the patients display the phenotype of classical MD, but milder phenotypes with longer survival are observed in about $6 \%$ of the patients, and a further $3 \%$ have the mildest form, OHS. ${ }^{4}$ Until now only 13 patients with OHS have been reported (www.hgmd.org).

To date, close to 270 mutations in the ATP7A gene are registered in the Human Mutation Database (www.hgmd.org). No deep intronic mutations have so far been reported in the literature. Traditional mutation screening including investigation of exon deletions or duplications using multiplex ligation-dependent probe amplification detects $~ 95-98 \%$ of the mutations. Identification of the last $2-5 \%$ of the mutations requires extra effort.

Here, we describe, for the first time, the identification of three mutations located in deep intronic sequences, leading to the activation of ATP7A pseudo-exons. The mutations were identified after detailed analyses of ATP7A mRNA from three unrelated patients with different phenotypes. Two of the patients, P1 and P3, exhibit the mildest allelic form OHS, whereas the third patient, P2, exhibits classical MD but with prolonged survival.

\section{MATERIALS AND METHODS}

\section{Patients}

Patient P1 and patient P3 had OHS. Both were 17 years old, were intellectually normal and had occipital horns. Patient P1 has been described previously. ${ }^{5}$ He had severe feeding difficulties in childhood, resulting in failure to thrive. He presented with kinky hair, hyperextensible joints, hypotonia, recurring bilateral inguinal hernia, bladder infections and symptoms of dysautonomia (chronic diarrhea, fainting spells and orthostatic hypotension). On X-ray examinations, severe osteopenia and short claviculae with widening of lateral parts (hammer shape) were noted. He had bilateral hip dislocation and minimal occipital horns. He presented walking difficulties from childhood and wore bilateral leg braces. At the age of 10 years, severe feeding problems persisted and tube feeding during the night was introduced. In the following years, his condition further deteriorated. At the age of 17 years, he became ill at school with severe abdominal pain, vomiting and diarrhea. He was taken to a

${ }^{1}$ Applied Human Molecular Genetics, Kennedy Center, Copenhagen University Hospital, Rigshospitalet, GI. Landevej 7, Glostrup, Denmark; ${ }^{2}$ Center for Medical Genetics, Ghent University, Ghent, Belgium; ${ }^{3}$ Department of medical genetics, Oslo University Hospital, National Hospital, Oslo, Norway; ${ }^{4}$ Centro de Genética Preditiva e Preventiva, Instituto de Biologia Molecular e Celular, Universidade do Porto, Porto, Portugal; ${ }^{5}$ Trás-os-Montes e Alto Douro Hospital Center, Vila Real, Portugal

*Correspondence: LB Møller, Applied Human Molecular Genetics, Kennedy Center, Copenhagen University Hospital, Rigshospitalet, Gl. Landevej 72600 Glostrup, Denmark. Tel: + 454326 0100; Fax: + 454343 1130; E-mail: Lisbeth.Birk.Moeller@ regionh.dk

Received 25 April 2013; revised 22 July 2013; accepted 24 July 2013; published online 4 September 2013 
hospital. He had a bowel obstruction and was successfully treated for it He seemed fine. However, during the night, he developed breathing problems and stopped breathing completely. Efforts to stimulate his breathing failed and he died. P3 had a history of multiple urinary infections, bilateral inguinal recidivate hernia, mild mitral valve prolapse and chronic diarrhea. He had anterior rotation of the femoral neck, genu valgu, pes planus and progressive articular degeneration leading to difficulties in walking. He had joint limitation in extension range in both elbows and knees and had kyphoscoliosis.

P2 has the classical form of Menkes disease but with long survival as he died at the age of 10 years. In contrast to P1 and P3 he did not have skin laxity or hypermobile joints. At age 6 months he had an upper airway infection and then the first seizures were observed. A subdural hematoma was evacuated at the age of 2 years and frontal but no occipital bossing was described thereafter. EEG showed pronounced epileptic changes from the age of 6 months. CT scan showed pronounced changes frontally and in the area perfused by middle cerebral artery with cortical and central brain atrophy. He had Kinky hair (pili torti), which led to diagnosis, and light hypopigmented skin. He was severely mentally retarded, with basically no development after the age of 6 months. He did not react to vision stimuli and had never been able to say any words. He had never been able to stand or move around. At the age of 7 years he had a cardiac arrest but survived. He died at the age of 10 years from pneumonia.

\section{Copper uptake and retention in fibroblasts}

Fibroblast cell cultures obtained from 11 different patient-skin biopsies (and one control) were cultured as described previously. ${ }^{6}$ The copper uptake and retention profile of the fibroblast cultures was assessed as described previously. ${ }^{7}$

\section{Investigation of $A T P 7 A$ transcript}

Total RNA from $\sim 5 \times 10^{6}$ cultured skin fibroblasts was isolated with the RNAeasy kit (Qiagen, Bothell, WA, USA), and single-stranded complementary DNA (cDNA) was synthesized with the high-capacity cDNA Archive Kit in accordance with the manufacturer's instructions (Applied Biosystems, Foster City, CA, USA). In order to investigate for potential intronic located mutations, which might affect the splicing of ATP7A mRNA, we performed overlapping PCR on cDNA. Primer sequences can be obtained upon request. The PCR products were separated on a $1 \%$ agarose gel. PCR products having a different size compared with those obtained from the control sample were cut from the gel, purified and sequenced with the same primers, as used for the PCR amplification, using an ABI3100 sequencer (Applied Biosystems).

\section{Identification of the genomic mutations}

Using the genomic DNA from the three patients as template and the primer pairs: caacttcaccagcattgcat/cccgagtagctgggattaca for $\mathrm{P} 1$; agccattctgactggtgtga/ gtgggcaaagggcataaata for P2 and tgatcaatctttgttaccttgctt/ggaaatgcaaagcaaaccat for P3, the pseudo-exons and flanking regions were amplified by PCR, using HotStarTaq Plus DNA Polymerase (Qiagen Bothell). The products were subsequently sequenced with the same primers as those used for the PCR amplification, using an ABI3100 sequencer (Applied Biosystems).

\section{Quantitative RT-PCR}

Real-time PCR was performed on cDNA obtained from patient and control fibroblasts. A TaqMan 6-carboxy-fluorescein (FAM)-labeled probe and primer pair against the boundary between exon 1 and exon 2 (part number Hs00921963_ml) in the ATP7A cDNA was used to detect total amount of ATP7A transcript in all three patients. A probe and primer against the boundary between exons 10 and 11 (custom design) was used to detect the amount of wild-type transcript in P1, a probe and primer against the boundary between exons 14 and 15 (part number Hs00921957_m1) was used to detect the amount of wild-type transcript in P2 and a probe and primer against the boundary between exons 16 and 17 (part number Hs00921959_ml) was used to detect the amount of wild-type transcript in P3. A FAM-labeled probe and primers for the human GAPDH transcript (part number 4352934E) were used as an endogenous control. Relative quantification of GAPDH transcript was carried out on parallel samples. The cDNA samples obtained from the RNA preparations were assayed in triplicates using a concentration of $100 \mathrm{ng}$ per sample in a total volume of $25 \mu \mathrm{l}$. All probes were purchased from Applied Biosystems. PCR amplification and detection was performed with an ABI7500 (Applied Biosystems) in accordance with the manufacturer's instruction. The threshold cycle (CT) is defined as the fractional cycle number at which the fluorescence passes a fixed threshold. Standard curves of (CT) values compared with log cDNA concentration were prepared by assaying five-fold serial dilutions of control cDNA, from $100 \mathrm{ng}$ per sample to $0.16 \mathrm{ng}$ per sample, with the GAPDH and ATP7A probes, respectively. In all experiments, the amount of ATP7A and GAPDH mRNA was calculated by linear regression of the lines generated by the standard curves, log cDNA concentration against CT.

\section{RESULTS}

Of a cohort of 511 independent MD patients who have been referred to the Kennedy Center, no pathogenic variant was observed in 13 individuals by standard detection methods. Fibroblast cultures were available from 11 of these 13 patients. Reduced copper-excreting ability of fibroblasts from all the 11 patients, which is used as a diagnostic tool, indicated Menkes disease in all cases.

To test whether disease-causing mutations could be located in deep intronic sequences, we isolated RNA from these 11 fibroblast cultures and performed overlapping RT-PCR reactions encompassing the entire cDNA sequence of the ATP7A gene in the three patients and a healthy control in comparison.

Analysis of the RT-PCR products revealed an aberrant banding pattern in three patients: $\mathrm{P} 1, \mathrm{P} 2$ and $\mathrm{P} 3$. In the sample from patient P1, three bands were obtained by RT-PCR amplification of the fragment spanning from exons 9 to 13. One of the three products was of similar size as the product obtained in the control sample ( $\sim 600 \mathrm{bp}$ ), but a larger and a smaller product were also present. RT-PCR amplification of the fragment, spanning from exons 12 to 17 in samples from patients $\mathrm{P} 2$ and P3, revealed in both cases a larger band than the $\sim 800 \mathrm{bp}$ band obtained from the control sample (Figure 1).

Direct sequencing of the three PCR products from patient P1 revealed that the product of similar size as the product obtained from the control sample was in fact identical to the wild-type product. An insertion of $98 \mathrm{bp}$ was located at the junction between exons 10 and 11 in the larger product. The smaller product in patient P1 represents a transcript without exon 10. In addition, in the control sample, an alternative transcript without exon 10 was identified, which is in agreement with previous observations. ${ }^{8}$ The exon-10-skipping transcript appears to be present in a smaller amount in the healthy control compared with patient P1. Direct sequencing of the PCR products obtained from patients $\mathrm{P} 2$ and $\mathrm{P} 3$ revealed that an insertion of $69 \mathrm{bp}$ was located at the junction between exons 14 and 15 in patient $\mathrm{P} 2$, whereas an insertion of $68 \mathrm{bp}$ was located at the junction between exons 16 and 17 in patient P3.

The origin of the inserted sequences was determined by a Blast analysis (Basic Local alignment Search Tool; http://blast. ncbi.nlm.nih.gov/Blast.cgi) and corresponds perfectly to sequences located in intron 10, intron 14 and intron 16. The nomenclatures for the three insertions are (r.2406_2407ins2406+1015_2406+1112), (r.2916_2917ins2916+2411_2916+2479) and (r.3294_3295ins3294 +695_3294+ 762).

PCR amplification of the intronically located pseudo-exons and the flanking sequences on genomic DNA from the three patients followed by sequencing revealed the mutations c. $2406+1117 \mathrm{~A}>\mathrm{G}$ (IVS10+ $1117 \mathrm{~A}>\mathrm{G}$; g. $108533 \mathrm{~A}>\mathrm{G})$ in P1, c. $2916+2480 \mathrm{~T}>\mathrm{G}(\mathrm{IVS} 14+2480$ $\mathrm{T}>\mathrm{G} ; \mathrm{g} .117863 \mathrm{~T}>\mathrm{G})$ in $\mathrm{P} 2$ and c.3294 $+763 \mathrm{C}>\mathrm{G}(\mathrm{IVS} 16+763$ $\mathrm{C}>\mathrm{G}$; g.126650C $>\mathrm{G}$ ) in P3 (Table $1 \mathrm{~A}$ ). 

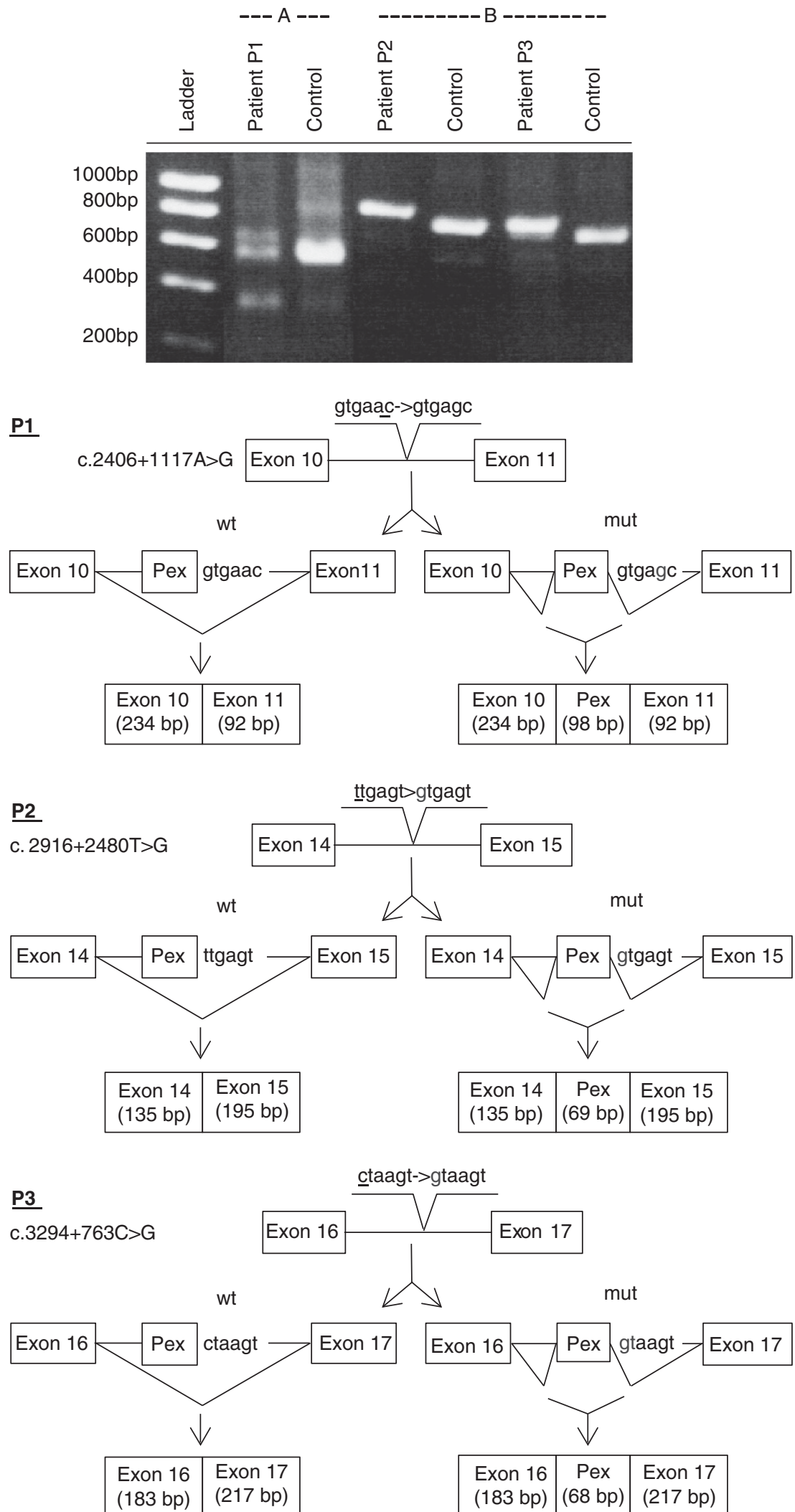

Figure 1 Deep intronic mutations leading to the inclusion of pseudo-exons in three patients. Total RNA isolated from cultured fibroblasts obtained from the three patients was investigated by RT-PCR. The ATP7A cDNA fragment from exons 9 to 13 was amplified in P1 and a control sample (A) using the primer pair tcattcttctatgttcctggagcgc/aagcagtgacccgttctggttaat and the cDNA fragment from exons 12 to 17 was amplified in P2 and P3 and control samples (B) using the primer pair aggaggcaaatttccagtgga/gtcctctatattccagttattc. The lower part of the figure shows a schematic illustration of the major effect of the wild-type (wt) and mutated (mut) intronic sequences with respect to inclusion of the pseudo-exons (Pex) in the final transcripts. 
The mutation designations are based on the NCBI reference sequence for ATP7A transcript NM_000052.5 and the genomic reference sequence NG_013224.1. The exon numbering is based on NG_013224.1.

The Human Splicing finder tool (http://www.umd.be/HSF/) (HSF) was used for in silico analysis of the $3^{\prime}$ acceptor and $5^{\prime}$ donor sites of the genomic regions encompassing the pseudo-exons. The $98 \mathrm{bp}$ pseudo-exon located in intron 10, the $69 \mathrm{bp}$ pseudo-exon located in intron 14 and the $68 \mathrm{bp}$ pseudo-exon located in intron 16 were investigated. The pseudo-exons were investigated, in the absence and presence of the mutation, respectively. This analysis revealed that the presence of the intronic mutation in all three cases increased the consensus value $(\mathrm{CV})$ of the cryptic donor splice site, leading to strong splice sites (Table 1A). The values increased from 78.80 to 90.96 (P1), from 66.30 to 93.14 (P2) and from 66.43 to 93.20 (P3), giving in all cases an increased CV of more than $10 \%$. Splice sites with CVs higher than 80 are strong splice sites, whereas splice sites with CV's between 65 and 70 are weak, as only few are active ${ }^{9}$ and a relative change in $\mathrm{CV}(\Delta \mathrm{CV})$ of $10 \%$ or more is likely to affect the splicing. ${ }^{10}$ These activated donor splice sites are all used in combination with high-score acceptor sites. Thus, the three mutations seem to activate pseudo-exons located in intron 10, intron 14 and intron 16 by the activation of new donor splice sites (Table 1A; Figure 1).

The insertion of the 98-bp pseudo-exon between exons 10 and 11 in patient $\mathrm{P} 1$ leads to a premature stop codon after eight aberrant amino acids. The resulting protein product is in this case only 810 amino-acid long in comparison with the wild type containing 1500 amino acids. The insertion of the 69-bp pseudo-exon between exons 14 and 15 in patient P2 leads to a premature stop codon after two aberrant amino acids. The resulting protein is predicted to contain 974 amino acids. The insertion of the 68-bp pseudo-exon between exons 16 exon 17 in patient P3 leads to a premature stop codon after 33 aberrant amino acids. The resulting protein is predicted to contain 1131 amino acids.

As ordinary RT-PCR (Figure 1) is not quantitative, real-time PCR using TaqMan probes was performed to determine whether the mutations lead to decreased amounts of ATP7A mRNA and also to determine the amount of wild-type transcript present. The relative total amount of ATP7A mRNA was measured using a TaqMan probe complementary to the junction sequence between exons 1 and 2 present in the transcripts in all three patients. In order to normalize to any differences in the cDNA input, a probe detecting the housekeeping gene, $G A P D H$, was used as an endogenous control

Table 1A Mutations located in introns and effects on the ATP7Atranscript

Mutation (underlined), with indication of pseudo-exon (in gray)

Intron 10: c.2406 + 1117A > G (r.2406_2407ins2406 + 1015_2406 + 1112)

tatatatgtcctttgcctgtgtgtttattgggttcatgttttcttattaatttatataa

tttcttcataaattatacctaataatacttttaaaatatttgtgtacttttag

gctgggcgcggtggctcatgcctgtaatcccagcgctttgggaggccgaggtgggtggatcacgag

gtcaggagtttgagaccggctggccaacatggtgaaccccatctctactaaaaatacaa

aaattagccaggcatggtggcgcgcgtctgtaatcccagctactcgggaggctgaggcag

Intron 14: c. 2916 + 2480T>G (r.2916 2917ins2916+2411_2916+2479)

agtatataagtgttcccttttctctgcatcgttgccaacatctgttatttttgatttt

ttaattatagccattctgactggtgtgaaggggtatttcattgtggtttaagttgcatt

tccctgatgattagtgatgttgagtatttttcatgtgttttttttatcacttgtatgt

cttcttttgagaggtgtctatttatgccetttgcccacttttaaatagggttgttttatc

Intron 16: c.3294 + 763C>G(r.3294_3295ins3294+695_3294 + 762

ttttcctgtcaggaaatgcagatctgcctcattgtttttaatggctgtattatactctat

agtgtgagtgtttgtttttttttgactttgcagtatatactcaacatccttatcagttt

ttgcaagtctgtctctacattaaatgcctatcaatgcaattgctaagtcaaaggatgcat

gcagttaaattttgatgaatgctaccaatacccatatgatgaatattaccaatatacatg
In silico prediction of splicing sites using HSF

Acceptor site: gtgtacttttagGC CV 81.32

Donor site, wt: ATGgtgaac CV 78.80

Donor site, mut: ATGgtgagc $\quad$ CV $90.96 \Delta \mathrm{CV}(\%)+15.43$

Acceptor site: $\quad$ ttttaattatagCC CV 80.93

Donor site, wt: ATGttgaat CV 66.30

Donor site, mut: ATGḡtgagt $\quad$ CV $93.14 \Delta \mathrm{CV}(\%)+40.48$

Acceptor site: tgacttttgcagTA CV 84.59

Donor site, wt: TTGctaagt CV 66.43

Donor site, mut: TTGğtaagt CV $93.20 \Delta \mathrm{CV}(\%)+40.30$

Detection of the genomic mutations and in silico prediction of activated splice sites. The nucleotides of the pseudo-exon located in intron 10 , intron 14 and intron 16 are highlighted in gray. The mutated nucleotides located in the introns, leading to the activation of the cryptic donor sites are underlined. The consensus value (CV) and the relative change in CV (\%) ( $\Delta \mathrm{CV}(\%))$ for the splicing sites obtained by HSF are listed.

Table 1B Expression of ATP7A transcript in patient fibroblasts

\begin{tabular}{|c|c|c|c|}
\hline Sample (ID) & $\begin{array}{c}\text { Total ATP } 7 A_{N} \text { transcript relative to unaffected } \\
\text { controls }{ }^{a} \text {. A TaqMan probe detecting all } \\
\text { transcript variants was used }\end{array}$ & $\begin{array}{l}\text { Wild-type } \text { ATP } A_{N} \text { transcript relative to unaf- } \\
\text { fected controls }{ }^{b} \text {. A TaqMan probe detecting } \\
\text { onlywild-type transcript was used }\end{array}$ & $\begin{array}{l}\text { ATP7A wild-type transcript, relative } \\
\text { to total ATP7A transcript in the } \\
\text { specific patient sample }\end{array}$ \\
\hline P1-94285 & $21.82 \pm 0.46$ & $12.83 \pm 1.15 \approx 13$ & 52.4 \\
\hline P2-9820 & $9.61 \pm 0.34$ & $0.19 \pm 0.10 \approx 0.2$ & 2.0 \\
\hline P3-95263 & $11.47 \pm 0.36$ & $1.77 \pm 0.09 \approx 2$ & 15.3 \\
\hline
\end{tabular}

Quantifications of total amounts of transcripts in the patient cells were performed in triplicates with the probe against the exon 1/exon 2 junction. Quantification of the amount of wild-type transcript in the P1, P2 and P3 cells was performed in triplicate using the probe against the exon 10/exon 11 junction, the exon 14 /exon 15 junction and against the exon $16 /$ exon 17 junction. In all experiments, the amount of ATP7A and GAPDH RNA was calculated by linear regression of the lines generated by the standard curves, log cDNA concentration against CT.

a Normalized ATP7AN value is calculated by dividing the ATP7A RNA value by the GAPDH RNA value (SDs are shown).

${ }^{b}$ ATP7A value relative to healthy control fibroblasts is calculated by dividing the normalized ATP7AN value from the patient by the average ATP7AN value obtained from healthy control fibroblasts (SDs are shown). 
(Table 1B). The total amount of transcript was estimated to be $22 \%$ in $\mathrm{P} 1,12 \%$ in $\mathrm{P} 3$ and $10 \%$ in $\mathrm{P} 2$ of the amount found in the healthy control sample (Table 1B). Furthermore, to test for the presence of wild-type ATP7A mRNA in the three patients, a probe recognizing the exon 10/exon 11 junction was used for $\mathrm{P} 1$, a probe recognizing the exon 14/exon 15 junction was used for $\mathrm{P} 2$ and a probe recognizing the exon 16/exon 17 junction was used for P3. The total amount of wild-type transcripts in P1, P3 and P2 was estimated to be $\sim 13,2$ and $0.2 \%$ of the amount found in the healthy control sample, respectively.

\section{DISCUSSION}

Eukaryotic genes, including ATP7A, contain several intronic sequences, which look like exons but are normally ignored and not included in the spliced mRNA transcripts. Pathological events leading to the inclusion of a pseudo-exon include strengthening of donor or acceptor splice site as a result of de novo mutations. Investigation of ATP7A transcript by overlapping PCR covering the entire coding sequence of the cDNA sequence was used to screen for mutations in the intronic sequences leading to inclusion of pseudo-exons in 11 patients, in whom no mutation had been detected with standard diagnostic tools. We identified mutations in the intronic sequences leading to the inclusion of pseudo-exons in three patients. This corresponds to a frequency of $<1 \%$ for the combined cohort of 501 independent Menkes patients with confirmed mutations in the ATP7A gene who had been referred to the Kennedy Center. No aberrant PCR amplification products were identified in eight of the investigated Menkes patients. As the investigation of inclusion of pseudo-exons was performed by screening of the ATP7A cDNA sequence, using overlapping PCR performed with 11 primer pairs leading to fragments of $400-800 \mathrm{bp}$, it is impossible to exclude inclusion of a very small pseudo-exon in any of the eight patients. A more likely possibility is, however, that they contain a mutation in the promoter or the $3^{\prime}$-UTR sequence.

The inclusion of a pseudo-exon in the three patients leads to premature termination codons after 810,974 and 1131 amino acids as a result of frameshift. None of these transcripts are expected to encode functional proteins and are probably degraded by the nonsensemediated decay (NMD) mechanism, ${ }^{11}$ in agreement with the decreased amount of ATP7A transcript measured in all three patients. The apparently relatively increased amount of exon-10skipped transcripts in $\mathrm{P} 1$ compared with the control is probably an effect of NMD of the transcript containing the pseudo-exon. The encoded proteins all lack a large fraction of the C-terminal part, and it is thus unlikely that they possess any residual activity.

Only the wild-type ATP7A transcripts, found to be present in small amounts in the patients, are expected to encode functional proteins. The presence of wild-type transcript in P1 (13\%) and P3 (2\%) might explain the mild OHS phenotype, even though only $2 \%$ is present in P3. This is in agreement with our previous finding where we demonstrated that as little as $2-5 \%$ of the level of correctly spliced mRNA found in unaffected individuals is sufficient to develop OHS in contrast to classical MD. ${ }^{12}$ The observed classical MD phenotype in
P2, expressing only $0.2 \%$ wild-type transcript, underscores this connection.

Although the largest fraction of mutations in the ATP7A gene is present in the coding regions, these findings of deep intronic mutations in three patients demonstrate that failed detection of mutations in the coding regions should be followed by RNA investigation. The activation of pseudo-exons is a rare event, but as in vivo correction of Menkes disease using antisense oligonucleotides (AON) against splice site junctions has been demonstrated in zebrafish, ${ }^{13}$ AON might in the future be able to rescue Menkes disease in patients with mutations leading to the activation of pseudo-exons.

In conclusion, we demonstrate for the first time pseudo-exon activations caused by mutations located in deep intronic sequence of the ATP7A gene.

\section{CONFLICT OF INTEREST}

The authors declare no conflict of interest.

\section{ACKNOWLEDGEMENTS}

We thank the Menkes families for participating in this investigation. Written informed consent is obtained from the relatives of the three patients. We would like to thank Gabriela Soares, Unidade Genética Médica, CGMJM, Praça Pedro Nunes, 88, 4099-028, Porto, Portugal for collaboration. We are also very grateful to Jette Rune Rasmussen for assistance with generating the figure and to Brage Storstein Andresen for critical review of this manuscript. The research leading to these results has received funding from the Lundbeck Foundation.

1 Menkes JH, Alter M, Steigleder GK, Weakley DR, Sung JH: A sex-linked recessive disorder with retardation of growth, peculiar hair and focal cerebral and cerebellar degeneration. Pediatrics 1962; 29: 764-779.

2 Kodama H, Murata Y: Molecular genetics and pathophysiology of Menkes disease. Pediatr Int 1999; 41: 430-435.

3 Das S, Levinson B, Vulpe C, Whitney S, Gitschier J, Packman S: Similar splicing mutations of the Menkes/mottled copper-transporting ATPase gene in occipital horn syndrome and the blotchy mouse. Am J Hum Genet 1995; 56: 570-576.

4 Møller LB, Mogensen M, Horn N: Molecular diagnosis of Menkes disease: genotypephenotype correlation. Biochimie 2009; 91: 1273-1277.

5 De Paepe A, Loeys B, Devrient K, Fryns JP: Occipital horn syndrome in a 2 year old boy. Clin Dysmorphol 1999; 8: 179-183.

6 Skjørringe T, Tümer Z, Møller LB: Splice site mutations in the ATP7A gene. PLoS One 2011; 6: e18599.

7 Møller LB, Hicks JD, Holmes CS et al: Diagnosis of copper transport disorders. Curr Protoc Hum Genet 2011; Chapter 17: Unit17.9.

8 Dierick HA, Ambrosini L, Spencer J, Glover TW, Mercer JFB: Molecular structure of the Menkes disease gene (ATP7A). Genomics 1995; 28: 462-469.

9 Desmet FO, Hamroun D, Lalande M, Collod-Beroud G, Claustres M, Béroud C: Human Splicing Finder: an online bioinformatics tool to predict splicing signals. Nuclei Acids Res 2009; 37: e67.

10 Mount SM: A catalogue of splice junction sequences. Nuclei Acids Res 1982; 10 : 459-472.

11 Maquat LE: When cells stop making sense: effects of nonsense codons on RNA metabolism in vertebrate cells. RNA 1995; 1 : 453-465.

12 Møller LB, Tümer Z, Lund C et al: Similar splicesite mutations of the ATP7A gene lead to different phenotypes: classical Menkes disease or occipital horn syndrome. Am J Hum Genet 2000; 66: 1211-1220.

13 Madsen EC, Morcos PA, Mendelsohn BA, Gitlin JD: In vivo correction of a Menkes disease model using antisense oligonucleotides. Proc Natl Acad SCi USA 2008; 105 . 3909-3914. 